\title{
How Groups of People Interact with each other on Twitter During Academic Conferences
}

Xidao Wen

School of Information Sciences

University of Pittsburgh

$135 \mathrm{~N}$. Bellefield Ave.

Pittsburgh PA 15260, USA

xiw55@pitt.edu

\section{Denis Parra}

Department of Computer Science

Pontificia Universidad Católica de Chile

Vicuña Mackenna 4860

Santiago, Chile

dparra@ing.puc.cl

\section{Christoph Trattner}

Know-Center

Graz University of Technology

Inffeldgasse 16c, A-8010 Graz, AT

ctrattner@know-center.at

Copyright is held by the author/owner(s).

CSCW'14, xxxxx, 2014, Baltimore, MD, USA.

ACM 978-1-4503-1889-1

\begin{abstract}
This paper shows a work-in-progress of a recently started project, which aims to understand how people interact with each other on Twitter during academic conferences, with emphasis on different user groups. As a first step in that direction, we manually classified the users of four conferences into five user groups and investigated with which other groups they communicate, how much they contribute to the Twitter stream and how much attention they receive from their peers.
\end{abstract}

\section{Author Keywords}

Twitter; Conferences; Scientific Communities

\section{ACM Classification Keywords}

H5.m. Information interfaces and presentation (e.g., HCI): Miscellaneous.

\section{Introduction}

Twitter is the most popular micro-blogging service in the western-world. In Twitter, people write messages no longer than 140 characters known as tweets. Users connect to each other by following other accounts, creating an information service and a social network [2]. While Twitter has been studied from many perspectives, research on Twitter in the context of 


\begin{tabular}{|l|l|l|l|l|}
\hline & $\begin{array}{l}\text { \#ht } \\
\mathbf{2 0 1 2}\end{array}$ & $\begin{array}{l}\text { \#umap } \\
\mathbf{2 0 1 2}\end{array}$ & $\begin{array}{l}\text { \#recsys } \\
\mathbf{2 0 1 2}\end{array}$ & $\begin{array}{l}\text { \#ectel } \\
\mathbf{2 0 1 2}\end{array}$ \\
\hline Dates captured & $\begin{array}{l}\text { June } \\
24-28\end{array}$ & $\begin{array}{l}\text { July } \\
16-20\end{array}$ & $\begin{array}{l}\text { Sept. } \\
10-13\end{array}$ & $\begin{array}{l}\text { Sept. } \\
18-21\end{array}$ \\
\hline \# Twitter users & 61 & 51 & 266 & 91 \\
\hline \# Total tweets & 254 & 234 & 2022 & 434 \\
\hline a) \# Mentions & 24 & 32 & 265 & 17 \\
\hline b) \# Replies to & 19 & 16 & 60 & 138 \\
\hline c) \# Re-tweets (RT) & 105 & 104 & 1087 & 38 \\
\hline $\begin{array}{l}\text { d) \# Isolated tweets: } \\
\text { not a), b), c) }\end{array}$ & 106 & 82 & 610 & 241 \\
\hline $\begin{array}{l}\text { \% Users re-tweeted, } \\
\text { mentioned, replied-to }\end{array}$ & $34.4 \%$ & $37.3 \%$ & $34.6 \%$ & $46.2 \%$ \\
\hline \# Faculty & 19 & 23 & 61 & 51 \\
\hline \# Industry & 16 & 7 & 120 & 17 \\
\hline \# Junior Researcher & 6 & 3 & 6 & 3 \\
\hline \# Organization & 4 & 8 & 19 & 11 \\
\hline \# Senior Researcher & 15 & 18 & 53 & 15 \\
\hline
\end{tabular}

Table 1. Description of the users' groups and associated Twitter activity

academic conferences is rather rare $[1,3]$. To contribute to this sparse strand of research, we present in this paper preliminary results of a recently started project, which aims to understand how people, and in particular different kinds of user groups, interact with each other on Twitter during academic conferences. As a first step in that direction, we have analyzed the Twitter data of four different computer science conferences, which took place in 2012. To that end, we manually classified the users into five different groups: Junior Researcher, Senior Researcher, Faculty, Industry or Organization. As our first analyses reveal, the activity between conferences and among groups varies to a great extent, but is still possible to find some generalizations. We observe clear homophily between certain groups, i.e., people mainly interact with peers with similar experience in the community. This observation might seem obvious, but it has an important implication: in particular, newcomers' participation is scarce in three out of four conferences. It is even scarcer in the attention they receive from the conference community, challenging the hypothesis of Reinhardt et al. [4] who argue that Twitter is especially beneficial for newcomers to become engaged with the researcher community.

\section{Data Set \& Collection Process}

To obtain the data we collected Tweets available over the Twitter API by searching for the corresponding conference-hashtag. The particular conferences we targeted were Hypertext 2012 (\#ht2012), UMAP 2012 (\#umap2012), RecSys 2012 (\#recsys2012), and ECTEL 2012 (\# ectel2012). Table 1 shows the statistics of all four datasets. \# recsys2012 was the largest in terms of users and number of tweets generated. However, \# ectel2012 stands over the rest in terms of the most engaged community, indicated by the percentage of users re-tweeted, mentioned or replied-to. We also show in Table 1 the number of tweets produced by the five groups of users, groups traditionally present at an academic event. We manually classified the users using various Web-based sources: social (Twitter data) and Non-Social (DBLP and personal web page). The classification rules were as follows: a) Junior Researcher (J): Master's and PhD students in their first year, who have never published previously in the conference; b) Senior Researcher (SR): PhD students and PhDs who have published at least once in the conference. We also included postdocs in this group; $c$ ) Faculty $(F)$ : professors, lecturers and senior scientists with prior experience and participation in the community; d) Industry (I): accounts of people who work in the industry; and e) Organizations $(O)$ : This group of Twitter users represents organizations such as the ACM or IEEE, usual conference organization, etc. 


\begin{tabular}{|c|c|c|c|c|c|c|c|c|c|c|c|c|}
\hline \multirow[b]{2}{*}{ Category } & \multicolumn{3}{|c|}{ HT12 } & \multicolumn{3}{|c|}{ UMAP12 } & \multicolumn{3}{|c|}{ RECSYS12 } & \multicolumn{3}{|c|}{ ECTEL12 } \\
\hline & Attention & Contribution & $\mathrm{CR}$ & Attention & Contribution & $\mathrm{CR}$ & Attention & Contribution & $\mathrm{CR}$ & Attention & Contribution & $\mathrm{CR}$ \\
\hline $\mathbf{F}$ & 3.05 & 4.00 & 0.76 & 4.00 & 6.19 & 0.65 & 6.15 & 8.31 & 0.74 & 2.65 & 5.60 & 0.47 \\
\hline SR & 1.87 & 2.87 & 0.65 & 4.33 & 5.61 & 0.77 & 7.68 & 12.64 & 0.61 & 2.00 & 5.00 & 0.40 \\
\hline $\mathbf{J R}$ & 4.17 & 5.83 & 0.71 & 0.00 & 1.67 & 0.00 & 2.17 & 5.33 & 0.41 & 0.00 & 3.67 & 0.00 \\
\hline 0 & 6.75 & 13.50 & 0.50 & 0.50 & 2.00 & 0.25 & 1.11 & 3.95 & 0.28 & 1.82 & 3.91 & 0.47 \\
\hline I & 0.63 & 2.81 & 0.22 & 0.86 & 1.86 & 0.46 & 4.91 & 5.88 & 0.84 & 1.88 & 4.18 & 0.45 \\
\hline
\end{tabular}

Table 2. Average user Attention (A), Contribution (C) and Conversion Rate ( $C R=A / C)$ for each group at each conference.

\section{Results}

To understand each group's activity, we calculated three metrics per group at each conference, as shown in Table 2. We first analyzed the posts of each group to the Twitter stream using the conference hashtag. We calculated this group contribution $(\mathrm{C})$ by counting the number of messages tweeted and normalizing by group size. Then, we measured the attention ( $A$ ) received by a group by identifying the posts where their members were mentioned, replied-to, or re-tweeted. We also calculated the conversion ratio between these two measures $(C R=\mathrm{A} / \mathrm{C})$. We highlight three results: (1) No group has a $C R$ larger than 1 , i.e., no group received more attention compared to what they contributed. (2) Groups well established in a community such as $\mathrm{F}$ and SR had decent conversion rates across conferences, unlike the other groups for which $C R$ varies greatly, and (3) the JR group received little or no attention with the sole exception of the HT12 conference.

We also analyzed the interaction among groups. To this end, we calculated the proportion of conversational tweets produced by a group, which were directed to members of the other groups, as shown in Table 3. The table shows signs of homophily between the more experienced groups in each community ( $F$ and $S R$ ), which, in addition, received the largest attention from other groups with the exception of RecSys12, where accounts in the industry category (I) received more attention. In general (with the exception of HT12 for JR and ECTEL12 for $\mathrm{O}$ ) JR and $\mathrm{O}$ groups received very little attention of the more established $F$ and SR groups. Although these results are rather expected, it hinders the potential of Twitter as a channel that can increase newcomers' contributions in a research community.

\section{Conclusions \& Future Work}

In this work we show preliminary results of a recently started project, which aims at understanding how people or groups of people interact on Twitter, especially in academic conferences. We classified the users of four conferences into five groups and investigated their communication in terms of contribution and attention across groups. One limitation of our work is the manual classification of users. 


\begin{tabular}{|c|c|c|c|c|c|c|c|c|c|c|c|c|c|c|c|c|c|c|c|c|}
\hline \multirow[b]{2}{*}{$\mathbf{F} \backslash \mathbf{T}$} & \multicolumn{5}{|c|}{ HT12 } & \multicolumn{5}{|c|}{ UMAP12 } & \multicolumn{5}{|c|}{ RECSYS12 } & \multicolumn{5}{|c|}{ ECTEL12 } \\
\hline & $\mathbf{F}$ & SR & $\mathbf{J R}$ & $\mathbf{0}$ & I & $\mathbf{F}$ & SR & JR & 0 & I & $\mathbf{F}$ & SR & JR & 0 & I & $\mathbf{F}$ & SR & JR & 0 & I \\
\hline $\mathbf{F}$ & 0.43 & 0.16 & 0.20 & 0.16 & 0.05 & 0.53 & 0.42 & 0.00 & 0.02 & 0.04 & 0.36 & 0.30 & 0.01 & 0.00 & 0.34 & 0.73 & 0.14 & 0.00 & 0.02 & 0.11 \\
\hline SR & 0.46 & 0.19 & 0.15 & 0.12 & 0.08 & 0.32 & 0.60 & 0.00 & 0.01 & 0.06 & 0.22 & 0.33 & 0.01 & 0.02 & 0.42 & 0.42 & 0.13 & 0.00 & 0.16 & 0.29 \\
\hline JR & 0.52 & 0.00 & 0.12 & 0.20 & 0.16 & 0.40 & 0.60 & 0.00 & 0.00 & 0.00 & 0.21 & 0.38 & 0.08 & 0.00 & 0.33 & 1.00 & 0.00 & 0.00 & 0.00 & 0.00 \\
\hline 0 & 0.26 & 0.30 & 0.15 & 0.26 & 0.04 & 0.50 & 0.40 & 0.00 & 0.10 & 0.00 & 0.15 & 0.26 & 0.02 & 0.08 & 0.49 & 0.20 & 0.20 & 0.00 & 0.27 & 0.33 \\
\hline I & 0.27 & 0.31 & 0.19 & 0.19 & 0.04 & 0.42 & 0.50 & 0.00 & 0.08 & 0.00 & 0.26 & 0.25 & 0.00 & 0.02 & 0.47 & 0.58 & 0.20 & 0.00 & 0.13 & 0.10 \\
\hline
\end{tabular}

Table 3. Proportion of tweets directed between groups; e.g., group $F$ sent $16 \%$ of their conversational tweets to group SR in HT 2012.

Although it is not a trivial task, we plan to develop an automatic user classification based on the users' experience and engagement with the research community using different Web-based sources.

Considering the easy accessibility of the Twitter service we were expecting a larger participation of newcomers, but the data indicate, in general, a different picture. In order to draw a more concrete conclusion, we plan to collect data from more conferences and from different domains. In this way, we can generalize our results as well as investigate mechanisms that improve newcomers' participation. Moreover, we will utilize SNA (Social Network Analysis) metrics to understand whether the activity is dominated by a few people within groups, for instance, by analyzing each group's in- and out- degree distributions. Investigating the content shared by different groups is also among our goals; recently developed algorithms allow using topic modeling on short pieces of text to unveil latent topics among Twitter conversations. In the context of the conference events, we want to study which talks during the conference produce more buzz and which groups or users tend to originate this micro-blogging activity.
Acknowledgments: We acknowledge the valuable feedback provided by Prof. YuRu Lin from University of Pittsburgh. This work is partially supported by the Know-Center at Graz University of Technology.

\section{References}

[1] Ebner, M., Altmann, T. and Selver, S.: @twitter analysis of \# edmedia10- is the \#informationstream usable for the \#mass. - in: Form@re [Elektronische Ressource] 74, 1 - 11, 2011.

[2] Kwak, H., Lee, C., Park, H. and Moon, S.: What is Twitter, a social network or a news media?. In

Proceedings of the 19th international conference on World wide web (WWW '10). ACM, New York, NY, USA, 591-600, 2010.

[3] Letierce, J., Passant, A., Decker, S., and Breslin, J. (2010). Understanding how Twitter is used to spread scientific messages. Presented at the Web Science Conference 2010, Raleigh, NC, USA, 2010.

[4] Reinhardt, W., Ebner, M., Beham, G. and Costa, C.: How People are Using Twitter during Conferences, Hornung-Prähauser, V., Luckmann, M. (Ed.), 5th EduMedia conference, Salzburg, 145-156, 2009. 